\title{
Factores Determinantes del Bienestar Financiero y su Relación con la Calidad de Vida en una Muestra de Profesionales de Guayaquil, Ecuador
}

\author{
Elías S. Ortiz ${ }^{1 \star}$, Germán Lobos ${ }^{2}$ y Daniel Guevara ${ }^{1}$ \\ (1) CENTRUM Católica Graduate Business School, Lima, Perú. Pontificia Universidad Católica del Perú, \\ Lima, Perú. Av. Universitaria 1801, San Miguel, Lima, Perú. (e-mail: eortizwom1@hotmail.com; \\ dguevaras@pucp.pe) \\ (2) Facultad de Economía y Negocios, Universidad de Talca, Chile. Av. Lircay s/n, Talca, Chile. 3465548 \\ Talca, Chile. (e-mail: globos@utalca.cl)
}

* Autor a quien debe ser dirigida la correspondencia.

Recibido May. 10, 2018; Aceptado Jul. 12, 2018; Versión final Ago. 13, 2018, Publicado Feb. 2019

\begin{abstract}
Resumen
El objetivo principal de este estudio, de corte transversal, fue identificar los factores determinantes del bienestar financiero y estimar su relación con la calidad de vida en una muestra de trabajadores profesionales de Guayaquil, Ecuador. Las escalas y subescalas utilizadas fueron: escala de satisfacción con la vida; escala de angustia/bienestar financiero; calidad de vida relacionada con la salud; escalas de depresión, ansiedad y estrés; y conducta hedonista. Se utilizaron coeficientes de correlación y modelos lineales generalizados para las estimaciones. Se obtuvo una correlación positiva y significativa entre satisfacción vital y bienestar financiero. Los factores determinantes del bienestar financiero fueron el ingreso, número de niños viviendo en el hogar, variables relacionadas con la salud física y mental, y conducta hedonista. La principal conclusión de este estudio es que los resultados pueden servir como evidencia para el diseño de políticas públicas locales que contribuyan al bienestar financiero de los trabajadores profesionales de Guayaquil.
\end{abstract}

Palabras clave: bienestar financiero; calidad de vida; trabajadores; conducta hedonista; políticas públicas

\section{Determining Factors of Financial Well-being and its Relation to Quality of Life in a Sample of Professional Workers of Guayaquil, Ecuador}

\begin{abstract}
The main aim of this cross sectional study was to identify the determining factors of financial well-being and to consider its relation to quality of life in a sample of professional workers of Guayaquil, Ecuador. The scales and subscales used in thee study were: satisfaction with life scale, incharge financial distress/financial wellbeing scale, health-related quality of life, depression, anxiety and stress scales, and hedonistic behavior. Correlation coefficients and generalized linear models were used for the estimations. A positive and significant correlation between life satisfaction and financial well-being was obtained. The determining factors of financial well-being were income, number of children living at home, variables related to physical and mental health, and hedonistic behavior. The main conclusion of this study is that the results can serve as evidence for the design of local public policies that contribute to workers' financial well-being.
\end{abstract}

Keywords: financial well-being; quality of life; workers; hedonistic behavior; public policies 


\section{INTRODUCCIÓN}

El enfoque causa-efecto supone que la calidad de vida (CV) global de una persona depende de su satisfacción en muchas áreas concretas de la vida (Loewe et al., 2014). Tales áreas pueden ser clasificadas en amplios dominios de la vida como salud, relaciones sociales, familia y finanzas (Bowling y Gabriel, 2007), o en dominios más específicos como ocupación y educación (Bustamante et al., 2017). Siguiendo a Diener et al. (1985) el concepto de CV incluye dos componentes subjetivos: 1) la satisfacción con la vida, o satisfacción vital, es el componente cognitivo y, 2) la felicidad es considerada el componente afectivo. Esto implica que los conceptos de satisfacción con la vida y felicidad están interrelacionados pero no son exactamente lo mismo. Según Diener et al. (1985) la satisfacción con la vida es una evaluación global de la calidad de vida de una persona de acuerdo con ciertos criterios de satisfacción elegidos por la misma persona; tales criterios dependen de una comparación de las circunstancias con la que se cree que es una norma apropiada. Por otra parte, Lyubomirsky (2008) define la felicidad como una experiencia, satisfacción o bienestar positivo, combinado con la sensación de que la vida propia es buena, significativa y que vale la pena. En la figura 1 se muestra la relación entre los conceptos de CV global, satisfacción vital y felicidad.

Según Joo y Grable (2004), mientras que el bienestar financiero (BF) generalmente se entiende como un subconstructo de la $\mathrm{CV}$, aún no hay consenso sobre cuál es la mejor manera de medirlo. Algunos autores han propuesto medir el BF a través de ítems o reactivos individuales. Por ejemplo, Newman et al. (2008) propusieron un solo ítem para medir la satisfacción personal con la situación financiera, y O'Neill et al. (2005) para medir el efecto percibido de los problemas financieros en la salud. Mientras que otros autores han optado por medir el BF a través de un conjunto de reactivos, tales como Günay et al. (2014), Loewe et al. (2014), y Prawitz et al. (2006). Según Woodyard (2013) el BF es un concepto integral y multidimensional que incorpora cuatro elementos: 1) medidas objetivas de la situación financiera (como ingresos, patrimonio neto, liquidez, consumo, idoneidad de la vivienda), 2) satisfacción financiera, 3) comportamiento financiero (como gestión financiera, disponibilidad de ahorro de emergencia, gestión del crédito, plan de retiro, gestión de riesgos, planificación del patrimonio / herencia) y, 4) percepción financiera (actitud y conocimiento financiero objetivo), y que no se pueden evaluar con una sola medida.

Las percepciones financieras incluyen actitudes financieras y conocimiento financiero objetivo. Por lo tanto, como medida del BF, la satisfacción financiera, los comportamientos financieros o las percepciones financieras solo pueden utilizarse como un componente de una gama más amplia de dimensiones subjetivas y objetivas. Esto implica que la satisfacción financiera, como medida subjetiva, es un indicador de BF, el cual es un componente importante de la satisfacción global con la vida (Xiao y Porto, 2016). Por ejemplo, la satisfacción financiera es uno de los ocho ítems subjetivos de la escala de angustia financiera / bienestar financiero desarrollada por Prawitz et al. (2006). La literatura reporta consistentemente una relación positiva entre ingreso y satisfacción financiera (Delafrooz y Paim, 2011; Joo y Grable, 2004; Grable et al., 2013; Hansen et al., 2008; Plagnol, 2011). Esto implica que aquellos individuos con más ingresos reportan un mayor nivel de satisfacción financiera, mientras que aquellos que tienen menos ingresos son más propensos a reportar una menor satisfacción financiera. Hansen et al. (2008) y Plagnol (2011) concluyeron que las personas de más edad son más propensas a reportar una mayor satisfacción financiera pero hasta un cierto límite, lo cual sugiere una U-invertida para aquellos grupos de más edad. Sin embargo, Delafrooz y Paim (2011) reportaron la edad como variable no significativa para explicar el BF.

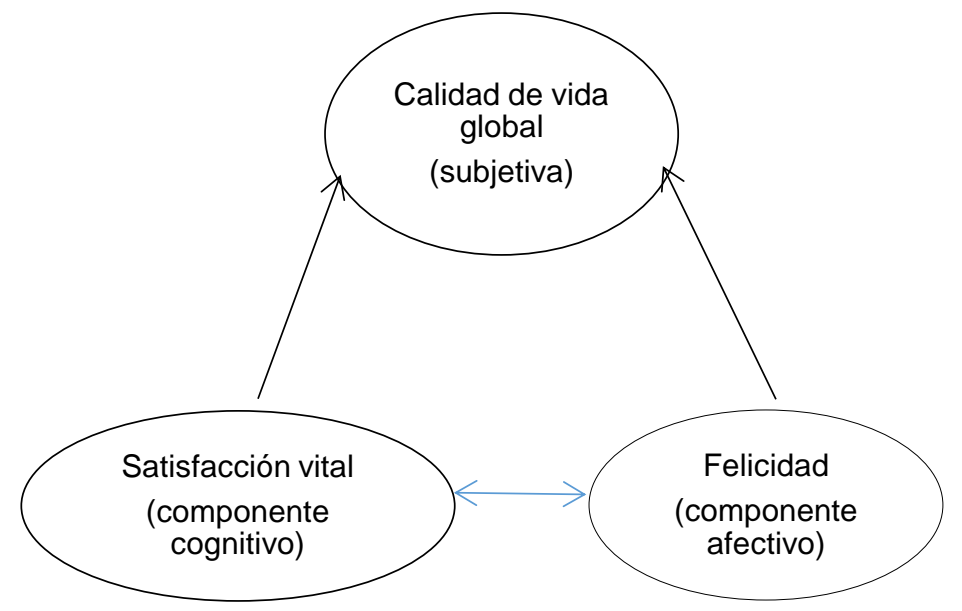

Fig. 1: Relación entre calidad de vida global, satisfacción vital y felicidad. 
La presencia de niños en el hogar también parece estar estrechamente relacionada con la satisfacción financiera (Van Praag et al, 2003; Ferrer-i-Carbonell, 2005). El mayor número de integrantes del hogar generalmente se ha relacionado con una menor satisfacción financiera, aunque algunos estudios han encontrado que, a diferencia del número de adultos, el número de hijos dependientes se asocia con una mayor satisfacción con la vida (Ferrer-i-Carbonell y Frijters, 2004). Barnard et al. (2014), Joo y Grable (2004), Lyons y Yilmazert (2005), y Delafrooz y Paim (2011) reportaron una relación entre satisfacción financiera y variables relacionadas con la educación y el estrés financiero (como indicador de salud mental). Joo y Grable (2004), y Delafrooz y Paim (2011) también incluyeron como variables explicativas el género y estatus marital. Más específicamente, Raccanello y Herrera (2014) plantean que las variables más relevantes para mejorar el bienestar financiero y la productividad de los trabajadores son la educación e inclusión financiera.

Este estudio contribuye a generar evidencia científica sobre los factores determinantes del BF y su relación con la CV en tres formas principales. Primero, determina las variables predictoras del BF en una muestra de trabajadores con educación formal (tercer nivel o cuarto nivel). Segundo, relaciona el BF con la CV aportando insumos para el diseño de políticas públicas por parte de los encargados de dichas políticas (policy-makers). Tercero, el alcance de este estudio es del tipo correlacional. Ello porque el estudio busca determinar relaciones entre BF y CV, además de encontrar explicaciones respecto del BF. Así, el objetivo principal de este estudio fue identificar los factores determinantes del BF y estimar su relación con la CV en una muestra de trabajadores profesionales de Guayaquil.

Lo anterior constituye el aspecto central de este trabajo y ahí radica su principal aporte. Esto porque en una sociedad de consumo la CV se convierte en un aspecto importante en la agenda de los policy-makers. Considerando que la CV de los trabajadores está recibiendo cada vez más atención, el diseño de dichas políticas públicas requiere no sólo de indicadores objetivos sino que también de indicadores subjetivos, tal como sugiere Veenhoven (2008). Por lo tanto, la provisión de indicadores subjetivos es importante para considerar las necesidades y deseos de los trabajadores en el diseño de estrategias de intervención social por parte de un gobierno. En este trabajo se plantearon las siguientes dos hipótesis:

Hipótesis 1: El BF y la CV están positiva y significativamente correlacionados. Esto significa que un mayor nivel de BF está asociado con una mayor CV;

Hipótesis 2: El nivel de ingreso, número de niños viviendo en el hogar, variables relacionadas con la salud física y mental, y conducta hedonista son las principales variables predictoras del BF.

\section{METODOLOGÍA}

La metodología se presenta en cuatro subsecciones: la primera describe la muestra y el procedimiento; en la segunda se presentan las escalas de medición utilizadas; en la tercera se define el modelo teórico; y en la cuarta se muestra el análisis estadístico de los datos.

\section{Sujetos y Procedimiento}

En esta investigación de corte transversal se aplicó un cuestionario estructurado en forma personal a una muestra de 913 trabajadores profesionales de Guayaquil, Ecuador. El tamaño de la muestra se determinó mediante un análisis de poder usando el programa G*power 3.1 (Faul et al., 2009). Este análisis arrojó un tamaño mínimo de la muestra de 694 trabajadores considerando una probabilidad de error $(\alpha=0,05)$, tamaño del efecto $(T E=0,25)$, poder $(1-\beta=0,95)$ y una proporción igual de hombres $\left(N_{1}\right)$ y mujeres $\left(N_{2}\right)$, esto es $N_{2} / N_{1}$ $=1$ ). Por facilidad operativa, la extracción de la muestra fue mediante muestreo no probabilístico por conveniencia (bola de nieve). El cuestionario fue aplicado por encuestadores previamente entrenados entre julio y octubre de 2017. El criterio de inclusión para la muestra fueron los profesionales con título de tercer y/o cuarto nivel de ambos sexos, sin discapacidades físicas (funcionales) o mentales (demencia). Sólo fueron encuestados los trabajadores que voluntariamente aceptaron participar y se protegió el anonimato de los participantes.

\section{Escalas de Medición}

En este análisis todas las variables son medidas auto-reportadas. Como parte de un cuestionario de mayor alcance, que incluyó otras medidas/escalas de satisfacción económica, estado de las finanzas y características personales, los trabajadores reportaron sus niveles de satisfacción con la vida completando la escala de satisfacción con la vida, en inglés Satisfaction with Life Scale (SWLS). En este estudio se utilizó esta escala como variable proxy para medir la CV. La SWLS, desarrollada por Diener et al. (1985), es un instrumento de cinco ítems utilizado para medir juicios cognitivos globales de satisfacción con la propia vida agrupados en una única dimensión: 1 = "En muchos aspectos mi vida se acerca a mi ideal"; 2 = "Mis 
condiciones de vida son excelentes"; 3 = "Estoy satisfecho con mi vida"; 4 = "Hasta ahora he obtenido las cosas importantes que he deseado en mi vida"; y 5 = "Si pudiera vivir mi vida de nuevo, no cambiaría nada". Los encuestados debieron indicar su grado de acuerdo con el planteamiento usando una escala Likert de 7 puntos ( 1 = "totalmente en desacuerdo" a 7 = "totalmente de acuerdo"). En este estudio se usó la versión en español de la SWLS, la cual ha mostrado adecuados niveles de consistencia interna en estudios previos en Ecuador (Schnettler et al. 2017) y Chile (Lobos et al., 2017) con valores $\alpha=0,84$ en ambos casos. En la escala SWLS un mayor puntaje corresponde a mayores niveles de satisfacción vital.

La escala de angustia financiera / bienestar financiero, en inglés InCharge Financial Distress/Financial WellBeing Scale (DFW), fue desarrollada por Prawitz et al. (2006). La DFW es una escala auto-reportada que consta de ocho ítems y es una medida subjetiva de angustia (o estrés negativo) / bienestar financiero. La escala DFW entrega una puntuación que representa el promedio de las respuestas a ocho indicadores individuales; así el puntaje mide de forma válida y confiable el constructo latente de la angustia / bienestar financiero percibido. Por lo tanto, un puntaje promedio más alto implica un mayor BF, mientras que un puntaje promedio más bajo implica una mayor angustia financiera (o menor BF). Por lo tanto, en este estudio la referencia a DFW se hará solo como BF. La versión en español de la DFW fue usada en este estudio, la cual ha mostrado adecuados niveles de consistencia interna $(\alpha=0,96)$ en estudio previos (Prawitz et al., 2006).

La escala de calidad de vida relacionada con la salud, en inglés Health-related Quality of Life (HRQOL) fue desarrollada por Hennessy et al., en 1994; es un concepto multidimensional que incluye dominios relacionados con la salud física, mental, emocional y el funcionamiento social. El primer ítem (HRQOL1) mide la autopercepción de salud general con base en una evaluación personal de la salud actual o la resistencia a enfermedades. Se realizó la siguiente pregunta: “¿Cómo diría que es su salud en general?” con un puntaje total de 1 = "muy mala" a 5 = "excelente". El segundo ítem (HRQOL2) se refiere al estado de salud física durante los últimos 30 días y el tercer ítem (HRQOL3) explora el estado de la salud mental reciente. El cuarto ítem (HRQOL4) se refiere a las limitaciones para desarrollar actividades sociales durante los últimos 30 días. Dado que HRQOL2 es una medida global de los síntomas físicos recientes y HRQOL3 es una medida global de la angustia mental y emocional reciente, en este estudio se sumaron ambos ítems para obtener una estimación de los días no saludables en general de una persona, con un máximo lógico de 30 días. Esta nueva variable la denominamos "Unhealthy Days" (UD).

Las escalas DASS, en inglés Depression, Anxiety and Stress Scales (DASS), fue desarrollada por Lovibond y Lovibond (1995) para medir los constructos de la depresión y la ansiedad de la salud mental; además para abordar la dificultad de las medidas emocionales anteriores en la discriminación entre la ansiedad y la depresión (Tian et al., 2013). En este trabajo se usó la versión reducida de la DASS, conocida como DASS21. La DASS-21 contiene 21 ítems con cuatro alternativas de respuesta en formato Likert. El puntaje de la escala se mide como: 0 = "nada aplicable a mí", 1 = "aplicable a mí en algún grado, o una pequeña parte del tiempo", 2 = "aplicable a mí en un grado considerable, o una buena parte del tiempo", y 3 = "muy aplicable a mí, o aplicable la mayor parte del tiempo". La persona debe indicar en qué medida la frase describe lo que le pasó o sintió durante la última semana.

Se incluyó la escala de actitud hacia el endeudamiento (AHE) desarrollada por Denegri et al. (1999). Esta escala consta de 11 ítems en formato tipo Likert con cinco opciones de respuesta: $1=$ muy de acuerdo a $5=$ muy en desacuerdo. Según Denegri et al. (2012) la escala está compuesta por dos factores ortogonales denominados "actitud austera frente al endeudamiento" (o perfil austero, PAU) y "actitud hedonista frente al endeudamiento" (o perfil hedonista, PHE). Mientras la primera incluye actitudes de cautela y reserva frente al endeudamiento, la segunda incluye actitudes proclives a contraer deudas sin evaluar las consecuencias. La escala de actitud hacia el endeudamiento ha mostrado adecuados niveles de consistencia interna en estudios previos tanto a nivel exploratorio $(\alpha=0,6)$ como concluyente $(\mathrm{GFI}=0,98$ y RMSEA=0,06). Tal como sugiere la literatura (Denegri et al., 2012) en este estudio se usaron los ítems 1, 2 y 11 para PHE y los ítems 4, 6, 7, 8 y 9 para PAU. Los ítems 3, 5 y 10 no fueron considerados, como proponen Denegri et al. (2012).

El cuestionario también incluyó preguntas respecto al nivel de ingreso (en dólares por mes) usando cinco rangos: 1 = "menos de 500", 2 = "de 500 a 999", 3 = "de 1.000 a 1.499", 4 = "de 1.500 a $1.999,5$ = "2.000 o más". Además se incluyeron otras medidas socioedemográficas como edad (años), género ( 0 = "masculino", 1 = "femenino"), situación de vida, en inglés life situation (LS) (0 = "está solo", 1 = "está con alguien"), posgraduado ( 0 = "no", 1 = "sí"), niños viviendo en el hogar" (número), y cantidad de bienes en el hogar considerando una canasta de 10 bienes (ducha, TV color, refrigerador, lavadora, Internet, vehículo, computador, horno microonda, calefont u otro sistema de ducha caliente, servicio de TV cable o satelital, y cámara de video filmadora). 


\section{Análisis Estadístico}

Respecto del modelo planteado en este trabajo, se usaron modelos lineales generalizados, en inglés Generalized Linear Models (GLM). Los GLM son una extensión del modelo lineal continuo, en inglés Continuous Lineal Model (CLM). Siguiendo la terminología usada por Wu (2005), el CLM toma la forma:

$$
\mathrm{E}\left(\mathrm{Y}_{\mathrm{i}}\right)=\mu_{\mathrm{i}}, i=1, \ldots n
$$

En la ecuación (1) la variable dependiente, a variable aleatoria, $Y_{i}$ es una variable independiente que se distribuye Normal, con media $\mu_{\mathrm{i}}$ y varianza constante $\sigma^{2}$. En notación matricial se puede reescribir la ecuación (1) como:

$$
\mathrm{E}(\mathrm{Y})=\mu=\mathrm{X} \beta
$$

En la ecuación (2) $\mu$ es de $\mathrm{n} \times 1, \mathrm{X}$ es de $\mathrm{n} \times \mathrm{k}, \beta$ es de $\mathrm{k} \times 1$ y $\mathrm{k}$ es el número de parámetros no conocidos incluyendo el intercepto. En los GLM nosotros podemos relajar algunos supuestos con respecto al CLM. Primero, no se requiere que la variable dependiente sea necesariamente continua y tenga distribución Normal, puede ser categórica o limitada (es decir truncada o censurada). Segundo, la relación entre la variable dependiente y las variables independientes puede ser de naturaleza no lineal. Tercero, la varianza de los errores no necesariamente es la misma en todos los niveles de las variables independientes (se indica heterocedasticidad). De acuerdo a Wu (2005) la primera extensión del CLM implica reconocer que muchas propiedades deseables de la distribución Normal son compartidas por una clase más amplia de distribuciones, conocida como la "familia de distribuciones exponenciales". La segunda extensión proviene del desarrollo de algunas funciones de enlace no lineal que relacionan el componente aleatorio $\mu$ con el predictor lineal. Los GLM toman la forma:

$$
\mathrm{E}(\mathrm{Y})=\mu=\mathrm{g}^{-1}(\mathrm{X} \beta)
$$

En la ecuación (3) el elemento $g($.$) es la función de enlace. Los GLM están formados por tres componentes$ básicos: 1) El componente aleatorio: $Y$ es el vector aleatorio tal que $E(Y)=\mu$. Los elementos de $Y$ son independientes e idénticamente distribuidos, cuya función de distribución pertenece a la familia de distribuciones exponenciales, y puede distribuirse no-Normal, tal como las distribuciones binomial, gamma o Poisson. 2) El componente sistemático: $X \beta$ es la parte sistemática del modelo, mientras que el predictor lineal está dado por $\eta=X \beta$, donde $\eta$ es una combinación lineal de la variables independiente $X$ y el correspondiente parámetro $\beta$. 3) La función de enlace: Los componentes aleatorio y sistemático están conectados a través de la función de enlace, la cual relaciona el predictor lineal $\eta$ con el valor esperado de la variable respuesta, $E(Y / X)=\mu$, a través de la función de enlace $g(\mu)$. Esto implica que $\eta=g(\mu)=E(Y / X)$, donde $g($.$) es una función$ monotónica y diferenciable. Esto significa que el predictor lineal $X \beta$ afecta la variable dependiente $Y$ a través de alguna forma funcional de $g($.$) . Además la función de enlace g(\mu)$ es invertible, lo cual significa que $g^{-1}(\eta)$ $=\mu(\mathrm{Wu} 2005)$.

Para el análisis descriptivo se calcularon promedios y desviaciones estándar (D.E.) para variables cuantitativas, y frecuencias (\%) para variables cualitativas (Monteagudo et al., 2015). Para el análisis de fiabilidad de los resultados y validez de las escalas se usó el coeficiente alfa de Cronbach ( $\alpha$ ), el índice de Kaiser-Mayer-Olkin (KMO) y el porcentaje de varianza explicada. El a proporciona una estimación de qué tan bien un grupo de indicadores mide un constructo unidimensional. Así, un puntaje alto (cercano a 1) indica unidimensionalidad, un puntaje bajo (distante de 1) sugiere que los datos tienen una estructura multidimensional (Prawitz et al., 2006). Como medida de la fuerza de la asociación entre dos variables se utilizaron los coeficientes de correlación de Pearson $(r)$, Eta $(\eta)$ y $V$ de Cramer $(V)$. El primero se usó para medir la relación entre dos variables cuantitativas, el segundo para medir la relación entre una variable cuantitativa (o condicionada) y otra cualitativa (o condicionante), y el tercero para para medir la relación entre dos variables cualitativas.

Se usó la técnica del modelo lineal generalizado (Wu 2005) para las estimaciones. Después de probar distintas distribuciones (como Normal, Poisson, de Gauss inversa y Gamma) y funciones de enlace asociadas, en el modelo final generado asumimos que la variable dependiente (DFW) se distribuye como una Normal, donde la Identidad es la función de enlace. Para evaluar la bondad de ajuste del modelo generado se utilizó la devianza (D), o discrepancia, y el estadístico Chi-cuadrado de la razón de verosimilitud ( $\left.\chi^{2} R V\right)$. La devianza permite conocer la variabilidad explicada por el modelo comparando la devianza del modelo nulo con la devianza del modelo completo. El modelo nulo corresponde al modelo sólo con la intersección y el modelo completo corresponde al modelo generado. Un valor más alto del estadístico $\chi^{2} \mathrm{RV}$ implica que la contribución 
de la variable incorporada en cada paso es significativa. Finalmente, los datos se analizaron usando el programa SPSS (Statistical Package for Social Science) para Windows, v. 22.

\section{RESULTADOS}

Se contactó un total de 962 trabajadores profesionales de Guayaquil, Ecuador. La tasa de respuesta fue $94,9 \%$. De todos los participantes en la muestra analítica $(n=913)$, el $48 \%$ eran hombres y el $52 \%$ mujeres. Por lo tanto, aunque el tamaño de muestra mínimo requerido fue 694, se recopilaron datos de más participantes en función de la expectativa de datos faltantes o respuestas de error. El análisis post hoc sugirió que la potencia $(1-\beta)=0,98$ dada la probabilidad de error $\alpha$, tamaño de la muestra y tamaño del efecto.

Con respecto al análisis de fiabilidad para las escalas SWLS, DFW, HRQOL (ítems 2, 3 y 4), DASS-21 y perfil hedonista (PHE), como sub-dimensión de la escala AHE, se obtuvo evidencia de una aceptable $(\alpha>0,7)$, buena $(\alpha>0,8)$ o excelente $(\alpha>0,9)$ consistencia interna en todos los casos. Las escalas y sub-dimensiones mostraron validez de constructo en todos los casos (KMO >0,5). El análisis de componentes principales de los cinco ítems que midieron la SWLS reveló un solo factor el cual representa el 70,8\% de la varianza. Con respecto a la escala DFW el mismo análisis confirmó la existencia de un solo factor para todos los ítems con $49,5 \%$ de la varianza explicada (Tabla 1 ).

Tabla 1: Puntaje de componentes e indicadores de fiabilidad y validez de medidas de bienestar

\begin{tabular}{|c|c|c|c|c|c|}
\hline \multirow{2}{*}{ Medida } & \multicolumn{2}{|c|}{ Importancia } & \multirow{2}{*}{$\begin{array}{l}\text { Alfa de } \\
\text { Cronbach }\end{array}$} & \multirow{2}{*}{$K M O$} & \multirow{2}{*}{$\begin{array}{l}\% \text { de la } \\
\text { varianza } \\
\text { explicada }\end{array}$} \\
\hline & Media & D.E. & & & \\
\hline Ítems de la escala de satisfacción con la vida (SWLS) & & & 0,885 & 0,879 & 70,83 \\
\hline En muchos aspectos mi vida se acerca a mi ideal & 5,18 & 1,27 & & & \\
\hline Mis condiciones de vida son excelentes & 5,18 & 1,21 & & & \\
\hline $\begin{array}{l}\text { Hasta ahora he obtenido las cosas importantes que he } \\
\text { deseado en mi vida }\end{array}$ & 5,50 & 1,35 & & & \\
\hline $\begin{array}{l}\text { Hasta ahora he obtenido las cosas importantes que he } \\
\text { deseado en mi vida }\end{array}$ & 5,41 & 1,28 & & & \\
\hline Si pudiera vivir mi vida de nuevo, no cambiaría nada & 4,94 & 1,61 & & & \\
\hline Ítems de la escala de angustia / bienestar financiero (DFW) & & & 0,836 & 0,891 & 49,45 \\
\hline ¿Cómo siente usted que es su nivel de estrés hoy día? & 6,26 & 1,82 & & & \\
\hline Grado de satisfacción con su actual situación financiera & 6,34 & 2,28 & & & \\
\hline ¿Cómo siente usted que es su actual situación financiera? & 5,78 & 1,79 & & & \\
\hline $\begin{array}{l}\text { ¿Con qué frecuencia se preocupa respecto si es capaz de } \\
\text { cumplir con los gastos mensuales normales? }\end{array}$ & 5,50 & 2,06 & & & \\
\hline $\begin{array}{l}\text { ¿Cuánta seguridad tiene usted de que podría encontrar } \\
\text { dinero para pagar por una emergencia financiera que } \\
\text { cuesta alrededor de } 1.500 \text { dólares? }\end{array}$ & 5,99 & 2,48 & & & \\
\hline $\begin{array}{l}\text { ¿Con qué frecuencia le pasa esto a usted?: Quiere ir a } \\
\text { comer fuera de casa, ir al cine o hacer cualquier otra cosa, } \\
\text { pero no lo hace porque no puede darse ese lujo }\end{array}$ & 6,17 & 2,22 & & & \\
\hline $\begin{array}{l}\text { ¿Con qué frecuencia usted encuentra que es justo vivir al } \\
\text { día? }\end{array}$ & 5,72 & 2,44 & & & \\
\hline $\begin{array}{l}\text { ¿Cuán estresado se siente usted con sus finanzas } \\
\text { personales en general? }\end{array}$ & 6,25 & 1,81 & & & \\
\hline $\begin{array}{l}\text { Ítems de la escala de calidad de vida relacionada con la } \\
\text { salud (HRQOL) (ítems } 2,3 \text { y } 4 \text { ) }\end{array}$ & & & 0,723 & 0,673 & 65,02 \\
\hline $\begin{array}{l}\text { Ítems de las escalas de depresión, ansiedad y estrés } \\
\text { (DASS-21) }\end{array}$ & & & 0,928 & 0,955 & 52,28 \\
\hline $\begin{array}{l}\text { Sub-dimensión perfil hedonista (PHE) (ítems } 1,2 \text { y 11) de } \\
\text { la escala de actitud hacia el endeudamiento }\end{array}$ & & & 0,703 & 0,614 & 62,78 \\
\hline
\end{tabular}

La Tabla 2 presenta las definiciones y estadísticas descriptivas de las variables incluidas en el modelo. La edad promedio de los trabajadores de la muestra fue 34,6 años (D.E. = 9,7, rango = 20-76 años). El 58,7\% de los trabajadores estaba solo y el restante $41,3 \%$ estaba con alguien. El promedio de niños viviendo en el hogar fue 0,9 (D.E. = 1,1). La mayoría de los trabajadores entrevistados tenía estudios de tercer nivel (75,6\%). La amplia mayoría de los trabajadores recibía menos de 1.500 dólares por mes (69,4\%). En este estudio el puntaje promedio de SWLS fue 24,3 (D.E. = 8,7). No se observaron diferencias significativas entre el puntaje promedio de hombres y mujeres; sin embargo, los trabajadores que estaban solos reportaron una menor SWLS en comparación con los que estaban con alguien con base en el test $\mathrm{t}$ de comparación de medias $(\mathrm{t}=$ 
$-2,87 ; p<0,004)$. El puntaje promedio de DFW fue 6 (D.E. $=1,5$ ). No se observaron diferencias significativas entre el puntaje promedio de hombres y mujeres y tampoco entre los que estaban solos o estaban con alguien. El puntaje promedio de la DASS-21 fue 11,6 (D.E. = 9,5). En este caso no se observaron diferencias significativas entre el puntaje promedio de hombres y mujeres; sin embargo, los trabajadores que estaban solos reportaron mayores niveles de DASS en comparación con los que estaban con alguien $(t=2,53 ; p<$ 0,011). En general se observó una "buena" o "muy buena" autopercepción de salud $(84,5 \%)$. El promedio de UD fue 9,2. Finalmente, el puntaje promedio de PHE de la escala AHE fue 5,6 (D.E. = 2,4). En este caso las mujeres reportaron un perfil más hedonista que los hombres $(t=-2,64 ; p<0,009)$ y también los trabajadores que estaban solos en comparación con aquellos que estaban en una relación ( $t=2,12 ; p<0,035)$.

Tabla 2: Estadísticas descriptivas de variables utilizadas en los modelos de regresión

\begin{tabular}{|c|c|c|c|}
\hline Variable & Definición & $\begin{array}{l}\text { Media } 0 \\
\%\end{array}$ & D.E. \\
\hline Edad & En años (rango: 20-76) & 34,6 & 9,7 \\
\hline Sexo & Variable binaria $(0=$ masculino, $1=$ femenino $)$ & 48,0 & - \\
\hline Situación de vida (LS) & Variable binaria (0 = "está solo", 1 = "está con alguien") & 58,7 & - \\
\hline Niños viviendo en el hogar & Número (rango: 0 a 7 ) & 0.90 & 1,1 \\
\hline Bienes en el hogar & Número (rango: 0 a 10) & 1,6 & 1,7 \\
\hline \multirow{3}{*}{ Educación } & Educación en 2 niveles & & \\
\hline & $1=$ tercer nivel & 75,6 & - \\
\hline & $2=$ cuarto nivel & 24,4 & - \\
\hline \multirow{6}{*}{ Ingresos } & Rango en 5 niveles (dólares por mes) & & \\
\hline & $1=$ "menos de $500 "$ & 10,8 & - \\
\hline & 2 = "de 500 a 999" & 29,5 & - \\
\hline & $3=$ "de 1.000 a $1.499 "$ & 29,1 & - \\
\hline & 4 = "de 1.500 a $1.999 "$ & 14,9 & - \\
\hline & 5 = "2.000 o más" & 15,7 & - \\
\hline $\begin{array}{l}\text { Escala de satisfacción con } \\
\text { la vida (SWLS) }\end{array}$ & $\begin{array}{l}\text { Puntuación total de } 5 \text { ítems con base en una escala de } 1 \text { a } 7 \\
\text { puntos (rango: } 0 \text { a 35) }\end{array}$ & 24,3 & 8,7 \\
\hline $\begin{array}{l}\text { Escala de angustia / } \\
\text { bienestar financiero } \\
\text { (DFW) }\end{array}$ & $\begin{array}{l}\text { Puntuación promedio de } 8 \text { ítems con base en una escala de } 1 \text { a } \\
10 \text { puntos (rango: } 1,1 \text { a 10,9) }\end{array}$ & 6,0 & 1,5 \\
\hline \multirow{6}{*}{ Autopercepción de salud } & Rango en 5 niveles & & \\
\hline & 1 = "muy mala" & 0,3 & - \\
\hline & 2 = "regular" & 7,4 & - \\
\hline & 3 = "buena" & 38,8 & - \\
\hline & 4 = "muy buena" & 45,7 & - \\
\hline & $5=$ "excelente" & 7,8 & - \\
\hline \multicolumn{2}{|c|}{ ¿Por cuántos días durante los últimos 30 días su salud física no estuvo bien? } & 4,4 & 7,5 \\
\hline \multicolumn{2}{|c|}{ ¿Por cuántos días durante los últimos 30 días su salud mental no estuvo bien? } & 4,8 & 7,6 \\
\hline \multicolumn{2}{|c|}{ ¿Por cuántos días durante los últimos 30 días no pudo realizar actividades comunes? } & 2,3 & 5,9 \\
\hline $\begin{array}{l}\text { Escalas de depresión, } \\
\text { ansiedad y estrés (DASS- } \\
\text { 21) }\end{array}$ & $\begin{array}{l}\text { Puntuación total de } 21 \text { ítems con base en una escala Likert de } 7 \\
\text { puntos ( } 1 \text { = "totalmente en desacuerdo" a } 7 \text { = "totalmente de } \\
\text { acuerdo") (rango: } 0 \text { a 63) }\end{array}$ & 11,6 & 9,5 \\
\hline $\begin{array}{l}\text { Escala de actitud hacia el } \\
\text { endeudamiento perfil } \\
\text { hedonista (PHE) de la } \\
\text { escala AHE (ítems } 1,2 \text { y } \\
\text { 11) }\end{array}$ & $\begin{array}{l}\text { Puntuación total de } 3 \text { ítems con base en una escala Likert de } 7 \\
\text { puntos ( } 1 \text { = "totalmente en desacuerdo" a } 5=\text { "totalmente de } \\
\text { acuerdo") (rango: } 0 \text { a 12) }\end{array}$ & 5,6 & 2,4 \\
\hline
\end{tabular}

La correlación de Pearson entre SWLS y DFW fue $r=0,41(p<0,00)$. La correlación entre DFW y las principales variables incluidas en este estudio fueron: edad $(r=0,13 ; p<0,00)$, sexo $(\eta=0,06)$, situación de vida $(\eta=0,02)$, niños viviendo en el hogar $(r=-0,08 ; p<0,02)$, ingreso $(\eta=0,42)$, salud física y mental durante los últimos 30 días $(r=-0,14 ; p<0,00)$, DASS-21 $(r=-0,32 ; p<0,00)$, y conducta hedonista de la escala AHE $(r=-0,18 ; p<0,00)$. Previo a la obtención de los modelos de regresión, se obtuvo la matriz de correlaciones de las principales variables explicativas incluidas en los modelos, lo cual permitió identificar identificando diferentes grados de correlación. En la Tabla 3 los resultados para el coeficiente $\eta$ se muestran en negrita y para $\vee$ en cursiva. Además, para los valores de $r$ y $\vee$ se indica la significancia al nivel ${ }^{* *} p<0,010{ }^{*} p<0,05$. 
El modelo multivariado final generado para explicar el BF de los trabajadores reveló efectos significativos para ingresos, niños viviendo en el hogar, UD, DASS-21 y PHE de la escala AHE (Tabla 4). La D sugiere que el modelo es adecuado; $\chi^{2} \mathrm{RV}$ indica que las variables incluidas en el modelo como un todo son significativas y por lo tanto es un buen ajuste. En la Tabla 4 los asteriscos indican la significancia al nivel ${ }^{* *} p<0,010{ }^{*} p<0,05$. Edad, sexo, situación de vida, cantidad de bienes en el hogar, educación y autopercepción de salud no resultaron significativas en el modelo.

Tabla 3: Correlaciones entre las variables

\begin{tabular}{|l|c|c|c|c|c|c|c|c|c|}
\hline Variable & $(1)$ & $(2)$ & $(3)$ & $(4)$ & $(5)$ & $(6)$ & $(7)$ & $(8)$ & $(9)$ \\
\hline (1) Edad & 1 & 0,022 & 0,229 & $0,086^{\star *}$ & 0,344 & $0,171^{* *}$ & 0,034 & $-0,146^{\star *}$ & $-0,032$ \\
\hline (2) Sexo & & 1 & 0,049 & 0,013 & $0,167^{\star *}$ & 0,022 & 0,021 & 0,022 & 0,068 \\
\hline (3) LS & & & 1 & 0,229 & $0,232^{\star *}$ & 0,090 & 0,002 & 0,084 & 0,077 \\
\hline (4) Niños & & & & 1 & 0,063 & 0,033 & 0,005 & 0,023 & 0,003 \\
\hline (5) Ingresos & & & & & 1 & 0,256 & 0,144 & 0,277 & 0,123 \\
\hline (6) SWLS & & & & & & 1 & $-0,079^{*}$ & $-0,280^{\star *}$ & $-0,126^{\star *}$ \\
\hline (7) UD & & & & & & & 1 & $0,218^{\star *}$ & $-0,014$ \\
\hline (8) DASS-21 & & & & & & & & 1 & 0,022 \\
\hline (9) PHE & & & & & & & & & 1 \\
\hline
\end{tabular}

Tabla 4: Coeficientes y bondad de ajuste del modelo lineal generalizado (GLM) generado

\begin{tabular}{|c|c|c|c|c|}
\hline & \multicolumn{4}{|c|}{ Variable dependiente: angustia / bienestar financiero (DFW) } \\
\hline & Coeficiente & Sig. & D.E. & $\begin{array}{l}\text { Odds-ratio (OR) } \\
(95 \% \text { intervalo de } \\
\text { confianza) }\end{array}$ \\
\hline Constante & 7,825 & ** & 1,1495 & - \\
\hline \multicolumn{5}{|l|}{ Ingresos } \\
\hline 1 = "menos de $500 "$ & $-1,691$ & ** & 0,1725 & 0,184 \\
\hline 2 = "de 500 a 999" & $-1,230$ & ** & 0,1313 & 0,292 \\
\hline $3=$ "de 1.000 a $1.499 "$ & $-0,901$ & 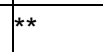 & 0,1312 & 0,406 \\
\hline 4 = "de 1.500 a $1.999 "$ & $-0,517$ & ** & 0,1509 & 0,596 \\
\hline 5 = "2.000 o más" & 0 & & . & 1 \\
\hline Niños viviendo en el hogar & $-0,086$ & * & 0,0388 & $0,918(0,853-0,992)$ \\
\hline UD & $-0,013$ & ** & 0,0057 & $0,987(0,974-0,992)$ \\
\hline DASS-21 & $-0,034$ & ** & 0,0047 & $0,967(0,960-0,978)$ \\
\hline PHE de la escala AHE & $-0,073$ & ** & 0,0178 & $0,930(0,903-0,959)$ \\
\hline Devianza (D) & \multicolumn{4}{|l|}{0,25} \\
\hline Chi-cuadrado de la razón de verosimilitud ( $\left.\chi^{2} \mathrm{RV}\right)$ & \multicolumn{4}{|c|}{$261,914^{\star *}(\mathrm{gl}=8)$} \\
\hline
\end{tabular}

\section{DISCUSIÓN}

Usando datos de un cuestionario administrado a 913 trabajadores profesionales de Guayaquil, Ecuador, se obtuvo información para probar las siguientes hipótesis: 1) el BF y la satisfacción con la vida están positiva y significativamente correlacionados y 2) el nivel de ingreso, número de niños viviendo en el hogar, variables relacionadas con la salud física y mental, y conducta hedonista son las principales variables predictoras del BF.

Con respecto a la primera hipótesis los resultados de este estudio muestran que el BF y la satisfacción con la vida (o componente cognitivo de la CV) están positiva y significativamente correlacionados. Esto significa que un mayor nivel de BF está asociado con una mayor satisfacción vital. Lo anterior es consistente con la literatura ya que el BF generalmente se entiende como un sub-constructo de la CV (Joo y Grable, 2004). Además, la CV depende de su satisfacción en muchas áreas concretas de la vida (Loewe et al., 2014), incluidas las finanzas (Bowling y Gabriel, 2007).

Con respecto a la segunda hipótesis los resultados muestran que el BF de los trabajadores profesionales de Guayaquil está relacionado con varias variables en múltiples ámbitos de la vida. El modelo de finanzas 
generado en este estudio confirma la relación positiva entre ingreso y BF, tal como sugieren diversos autores (Delafrooz y Paim, 2011; Joo y Grable, 2004; Grable et al., 2013; Hansen et al., 2008; Plagnol, 2011). Aun cuando Hansen et al. (2008) y Plagnol (2011) concluyeron que las personas de más edad son más propensas a reportar una mayor satisfacción financiera, en este estudio la edad no fue reportada como una variable predictora. Por lo tanto, nuestros resultados están en línea con lo reportado por Delafrooz y Paim (2011). Esto porque la edad podría estar contribuyendo indirectamente (a través de la variable ingreso) a explicar el BF. Aunque se observó una débil correlación entre edad e ingreso, la edad resultaba significativa cuando se excluía el ingreso del modelo. Sin embargo, la existencia de colinealidad entre ambas variables llevó finalmente a excluir la edad como variable explicativa. Lo anterior significa entonces que un mayor ingreso predice un mayor BF. Como reportaron los trabajos de Van Praag et al. (2003) y Ferrer-i-Carbonell (2005) la presencia de niños en el hogar está significativamente relacionada con el BF. Aunque los resultados de este trabajo mostraron un impacto negativo, en línea con lo informado por Ferrer-i-Carbonell (2005), pero diferente a lo obtenido por Van Praag et al. (2003). De acuerdo al modelo generado, el número de niños viviendo en hogar estaría asociado negativamente con el BF, lo cual podría explicarse porque para los trabajadores el mayor costo asociado al número de hijos no es posible de solventar dado el nivel de ingreso. Esto implica entonces un menor BF y por ende una menor CV. Cabe hacer presente que en este estudio no se observó una correlación significativa entre el número de niños viviendo en el hogar y la satisfacción con la vida, a diferencia de lo sugerido por Ferrer-i-Carbonell y Frijters (2004). Por lo tanto, con los resultados de este estudio se puede inferir que el número de niños viviendo en el hogar contribuye negativamente con la satisfacción con la vida pero indirectamente a través del BF, o variable de trasmisión.

El coeficiente negativo de las variables UD y DASS-21 sugiere que la salud física y mental durante los últimos 30 días y los niveles de depresión, ansiedad y estrés estarían contribuyendo negativamente a explicar el BF de los trabajadores. Estos resultados están en línea con lo reportado por Joo y Grable (2004), y Delafrooz y Paim (2011), quienes encontraron evidencia de asociación entre BF y salud mental. Nuestros resultados permiten inferir que un mayor número de días en que la salud física y mental no estuvo bien, así como mayores niveles de depresión, ansiedad y estrés estarían contribuyendo negativamente con el BF de los trabajadores. Los resultados de este estudio también permiten reportar evidencia de que una actitud hedonista implica un menor nivel de BF. Por lo tanto, las actitudes proclives a contraer deudas sin evaluar las consecuencias (Denegri et al., 2012) contribuyen negativamente al BF, y por lo tanto implican una menor CV. Los hallazgos de este estudio pueden servir para mejorar el diseño e implementación de las políticas públicas. Por ejemplo, fortalecer los servicios de salud física y mental de los trabajadores, así como fomentar una conducta más austera de los trabajadores.

Todo lo anterior se traduce en que al probar la hipótesis 1 (BF y CV positiva y significativamente correlacionados), y la hipótesis 2 (el nivel de ingreso, número de niños viviendo en el hogar, variables relacionadas con la salud física y mental, y conducta hedonista son las principales variables predictoras del BF) podemos concluir que el BF actúa como variable de transmisión entre sus propias variables predictoras (dadas por el modelo generado) y la CV (dada por la correlación significativa entre CV y BF). Esto significa que las variables independientes incluidas en el modelo contribuyen a explicar el BF; y este último actúa como variable de transmisión debido a su asociación significativa con la $\mathrm{CV}$, que en este trabajo la asimilamos al componente cognitivo de la CV global de una persona. Para lograr una actitud más racional hacia el endeudamiento podría sería relevante llevar a cabo una estrategia que mejore el acceso a la información y a los servicios financieros de los trabajadores, tal como sugieren Raccanello y Herrera (2014). La inclusión financiera es fundamental para las empresas porque permite optimizar la toma de decisiones financieras de los trabajadores, contribuyendo a mejorar la seguridad financiera y los índices de autopercepción de salud física y mental. Esto implica no sólo mayor productividad laboral sino que también un mayor BF de los trabajadores. Para el logro de lo anterior, y siguiendo la experiencia a nivel internacional, se podría implementar un programa de educación continua presencial en microfinanzas y administración del dinero, coordinado a través de las empresas y organizaciones de trabajadores. De manera no presencial la estrategia debería considerar la educación financiera a través de ambientes virtuales de aprendizaje, como educación a distancia mediante multimedia e Internet. Lo anterior está en línea con las relaciones entre satisfacción financiera y variables relacionadas con la educación y la salud reportadas por la literatura (Barnard et al., 2014; Joo y Grable, 2004; Lyons y Yilmazert, 2005; Delafrooz y Paim, 2011).

Por otra parte, desde el punto de vista de la política pública sobre inclusión financiera se podría evaluar el diseño e implementación de una plataforma on-line o e-learning. Finalmente, como recomendación para futuras investigaciones, parece conveniente determinar los niveles de patrimonio, gasto en consumo, ahorro e inversión, de manera de contar con un adecuado diagnóstico sobre la administración y planificación financiera de los trabajadores profesionales de Guayaquil.

Este estudio tiene algunas limitaciones metodológicas, lo que implica que los resultados deben interpretarse con precaución. Primero, este estudio reporta información sobre los patrones transversales de distintas 
dimensiones de la CV en una muestra de trabajadores, por lo que no podemos hacer inferencias con respecto a las tendencias en los últimos años. Segundo, en este estudio se entrevistó a trabajadores profesionales de Guayaquil, es decir, trabajadores con título de tercer o cuarto nivel, lo que implica que la muestra podría tener un sesgo de selección significativo. Tercero, en dos casos no se trabajó con las escalas completas. En el caso de la escala HRQOL se trabajó con la suma de dos ítems cuantitativos y en el caso de la escala AHE se trabajó con la sub-dimensión PHE, lo cual podría tener algún efecto sobre la validez de los resultados. Sin embargo, en ambos casos se validó con evidencia científica su utilización.

\section{CONCLUSIONES}

De acuerdo a los resultados de este estudio, y de su discusión y análisis se pueden obtener las siguientes tres conclusiones principales:

1) la correlación positiva y significativa entre el BF y la satisfacción con la vida (componente cognitivo de la CV) sugiere que los mayores niveles de BF están asociados con trabajadores más satisfechos con su vida;

2) dado que los factores predictores del BF fueron el ingreso, número de niños viviendo en el hogar, variables relacionadas con la salud física y mental, y la conducta hedonista, podría buscarse alternativas que contribuyan a mejorar los sistemas de salud física y mental, más allá de la responsabilidad individual de cada trabajador; además de fomentar una conducta más austera de los trabajadores profesionales de Guayaquil;

3) los resultados pueden servir como evidencia exploratoria para el diseño de políticas públicas locales o institucionales que promuevan la educación y la inclusión financiera de los trabajadores. Así, podría ser relevante el diseño de estrategias de intervención que incidan en el BF y la CV de los trabajadores profesionales de Guayaquil. Lo anterior a través de programas integrados de educación e inclusión financiera que incidan sobre la productividad laboral y el BF de los trabajadores. Esto es que consideren simultáneamente aspectos de salud y finanzas, o programas educativos y de sensibilización sobre microfinanzas y administración del dinero.

\section{REFERENCIAS}

Barnard, J., J. Fouché y W. Vosloo, Relationship between Financial Efficacy, Satisfaction with Remuneration and Personal Financial Well-Being, International Business \& Economics Research Journal, 13(6) 1455-1470 (2014)

Bowling, A. y Z. Gabriel, Lay Theories of Quality of Life in Older Age, Ageing Soc., 27 827-848 (2007)

Bustamante, M.A., M.C. Lapo, J.D. Torres y S.M. Camino, Factores Socioeconómicos de la Calidad de Vida de los Adultos Mayores en la Provincia de Guayas, Ecuador, Inf. Technol, 28(5), 165-176 (2017)

Delafrooz, N. y L.H. Laily Paim, Determinants of Financial Wellness among Malaysia Workers, Afr. J. Bus. Manage, 5(24) 10092-10100 (2011)

Denegri M., D. Cabezas, C. del Valle, Y. González y J. Sepúlveda, Escala de Actitudes hacia el Endeudamiento: Validez Factorial y Perfiles Actitudinales en Estudiantes Universitarios Chilenos, Univ. Psychol., 11(2) 497-509 (2012)

Denegri, M., M. Palavecinos, M. Ripoll y V. Yáñez, Caracterización Psicológica del Consumidor de la IX Región, In Consumir para Vivir y No Vivir para Consumir by M. Denegri, F. Fernández, R. Iturra, M. Palavecinos y M. Ripoll, pp. 731, Ediciones Universidad de La Frontera, Temuco, Chile (1999)

Diener, E., R. Emmons, R. Larsen y S. Griffin, The Satisfaction with Life Scale, J. Pers. Assess, 49 71-75 (1985)

Faul, F., E. Erdfelder, A. Buchner y A.G. Lang, Statistical Power Analyses using G*power 3.1: Tests for Correlation and Regression Analyses, Behav. Res. Methods, 41(4) 1149-1160 (2009)

Ferrer-i-Carbonell, A., Income and Well-being: An Empirical Analysis of the Comparison Income Effect, J. Public. Econ., 89, 997-1019 (2005)

Ferrer-i-Carbonell, A. y P. Frijters, How Important is Methodology for the Estimates of the Determinants of Happiness? Econ. J., 114 641-659 (2004)

Grable, J.E., S. Cupples, F. Fernatt y N. Anderson, Evaluating the Link between Perceived Income Adequacy and Financial Satisfaction: A Resource Deficit Hypothesis Approach. Soc Indic. Res, 114(3), 1109-1124 (2013)

Hansen, T., B. Slagsvold y T. Moum, Financial Satisfaction in Old Age: A Satisfaction Paradox or Result of Accumulated Wealth? Soc. Indic. Res., 89 323-347 (2008)

Hennessy, C., D. Moriarty, M. Zack, P. Scherr y R. Brackbill, Measuring Health-related Quality of Life for Public Health Surveillance, Public Health. Rep., 109 665-672 (1994)

Joo, S.H. y J.E. Grable, An exploratory framework of the determinants of financial satisfaction, J. Fam. Econ Issues, 25(1) $25-50$ (2004) 
Lobos, G., B. Schnettler, K.G. Grunert y C. Adasme, The Perceived Resources as Predictor on Food Satisfaction with Food-related Life among the Chilean Elderly: An Approach with Generalized Linear Models, J. Nutr.. Health Aging, 21(10) 1240-1249 (2017)

Loewe, N., M. Bagherzadeh, L. Araya-Castillo, C. Thieme y J.M. Batista-Foguet, Life Domain Satisfactions as Predictors of Overall Life Satisfaction among Workers: Evidence from Chile, Soc. Indic. Res, 118(1) 71-86 (2014)

Lovibond, S.H. y P.F. Lovibond, Manual for the Depression Anxiety \& Stress Scales, $2^{\mathrm{a}}$ Ed., Psychology Foundation, Sydney, Australia (1995)

Lyons, A. y T. Yilmazer, Health and Financial Strain: Evidence from the Survey of Consumer Finances, South Econ. J., 71(4), 873-890 (2005)

Lyubomirsky, S., The How of Happiness: A New Approach to Getting the Life You Want, The Penguin Press, New York, U.S.A. (2008)

Newman, C., L. Delaney y B. Nolan, A Dynamic Model of the Relationship between Income and Financial Satisfaction: Evidence from Ireland, Econ. Soc. Rev, 39(2) 105-130 (2008)

O'Neill, B., B. Sorhaindo, J.J. Xiao y E.T. Garman, Financial Distressed Consumers: Their Financial Practices, Financial Well-being, and Health, Financial Counseling and Planning, 16, 73-87 (2005)

Plagnol, A.C., Financial Satisfaction over the Life Course: The Influence of Assets and Liabilities, J. Econ. Psychol., 32(1) 45-64 (2011)

Prawitz, A.D., E.T. Garman, B. Sorhaindo, B. O'Neill, J. Kim y P. Drentea, InCharge Financial Distress/financial Well-being Scale: Development, Administration, and Score Interpretation, Financial Counseling and Planning, 17(1) 34-50 (2006)

Raccanello, K. y E. Herrera, Educación e Inclusión Financiera, Rev. Latinoam. Estud. Educ, XLIV (2) 119-141 (2014)

Schnettler, B., G. Lobos, M.C. Lapo, C. Adasme-Berríos y C. Hueche, Satisfaction with Life and Food-related Life in Ecuadorian Older Adults, Nutr Hosp, 34(1) 65-72 (2017)

Tian, P.S.O., S. Sawang, Y.W. Goh y F. Mukhtar, Using the Depression Anxiety Stress Scale 21 (DASS-21) across Cultures, Int. J. Psychol., 48(6) 1018-1029 (2013)

Van Praag, B.M.S., P. Frijters y A. Ferrer-i-Carbonel, The Anatomy of Subjective Well-being, J. Econ. Behav. Organ, 51 29-49 (2003)

Veenhoven, R., Effects of Happiness on Physical Health and the Consequences for Preventive Health Care, J. Happiness Stud, 9(3) 449-469 (2008)

Woodyard, A., Measuring Financial Wellness, Consumer Interests Annual, 59 1-6 (2013)

Wu, Z., Generalized Linear Models in Family Studies, J. Marriage Fam., 67 (4) 1029-1047 (2005)

Xiao, J.J. y N. Porto, Which Financial Advice Topics are Positively Associated with Financial Satisfaction? Journal of Financial Planning, 29(7) 52-60 (2016) 
